\title{
GENERATION OF NODAL CORE SIMULATOR UTILIZING VERA
}

\author{
Paul Turinsky ${ }^{1}$, Aaron Graham ${ }^{2}$, Hisham Sarsour ${ }^{3}$, Benjamin Collins ${ }^{2}$ \\ ${ }^{1}$ Statehouse, LLC \\ 618 North Boylan Avenue, Suite 724 \\ Raleigh, NC 27603 USA \\ ${ }^{2}$ Oak Ridge National Laboratory \\ P.O. Box 2008, Oak Ridge \\ TN 37831 USA \\ ${ }^{3} \mathrm{WSC}$, Inc. \\ 7196 Crestwood Blvd., Suite 300 \\ Frederick, MD 21703 USA
}

turinsky@ncsu.edu,grahamam@ornl.gov,SarsourH@ws-corp.com,collinsbs@ornl.gov

\begin{abstract}
Nuclear core simulators based upon few-group nodal diffusion method currently are widely used to predict light water reactor core behavior. Nodal parameters' input, e.g. cross-sections, discontinuity factors, and pin form factors, are typically generated utilizing lattice physics codes. In doing so, a number of approximations are introduced related to using zero current boundary conditions, 2-D radial geometry, and uniform thermal conditions in coolant and fuel. Usage of full core models with prediction fidelity typical of lattice physics to predict nodal parameters would eliminate these approximations. The VERA code can serve as such a full core model and was so utilized in this work. Via subsequent processing of VERA predictions, for a range of state points, nodal parameters and their functionalization in terms of coolant density, fuel temperature, and soluble poison concentration, were obtained and input to the NESTLE nodal code. By processing VERA predictions using consistent nodal methodologies as used in NESTLE, when using nodal parameters after functionalization based upon All-Rods-Out (ARO) VERA state points, the maximum reactivity and pin power differences between VERA and NESTLE were $2 \mathrm{pcm}$ and 0.003 for ARO core simulations. For rodded core simulations, these maximum differences grew to $58 \mathrm{pcm}$ and 0.04 . Increases in differences were determined to be attributed to usage of unrodded nodal parameters generated using the VERA ARO state points whether the core is partially rodded or not, consistent with lattice physics practice. Obtaining unrodded nodal parameters using the VERA rodded state points reduced maximum differences to $2 \mathrm{pcm}$ and 0.003 in pin powers.
\end{abstract}

KEYWORDS: nuclear core simulator, nodal cross-sections

\section{INTRODUCTION}

The objective of this work is to verify that a high-fidelity core simulator can be utilized to improve the fidelity of core simulators currently employed by the nuclear power industry for light water reactor 
(LWR) simulation. This is done for the neutronics portion of the core simulator, where current core simulators overwhelmingly employ a nodal methodology to computationally solve the few-group, coarse mesh, neutron diffusion equation in negligible wall clock time on personal computers.

Current practice is to employ a lattice physics code to generate the nodal parameters, i.e. few-group crosssections, discontinuity factors (DF), and pin form factors (PFF), used by the nodal core simulator. In doing so, one approximates radial and axial boundary conditions as zero current enabling 2-D radial single lattice models to be used. Thermal conditions for the coolant or fuel are treated as uniform. Also one approximates so called history effects. All these approximations not only impact the prediction at a stated exposure, but also predictions at future exposures through the spatial distribution of nuclei number densities. Various schemes have been developed to minimize the errors introduced by these approximations.

If high-fidelity core simulators could take the place of lattice physics codes, many of the approximations could be eliminated or substantially minimized. Work reported here utilizes the VERA high-fidelity core simulator [1] to provide the information to generate the nodal parameters, and the NESTLE nodal core simulator [2] to quantify the prediction agreement between VERA and NESTLE. Why a high-fidelity core simulator would not today be used to entirely replace a nodal core simulator is mainly due to two reasons: (1) the computational resources required in terms of number of processor cores and memory capacity, are much greater requiring access to such resources, and (2) the wall clock times are much greater (e.g. $\sim 1$ hour wall clock time using $\sim 1 \mathrm{~K}$ cores for a single state point of a 3 -D quarter core PWR model), which would noticeably impact how engineers function to complete nuclear core design and safety analysis, and prohibit usage in training simulators and core monitoring systems. These points would delay potential adoption of usage, thereby delaying the associated benefits. By contrast, if a highfidelity core simulator's usage was limited to mainly replacing a lattice physic code, the required amount of usage could be minimized with the bulk of required simulations done by a nodal core simulator. This would minimize the impact of reason (1) and completely address reason (2).

By way of brief introduction, the core simulator option of VERA, which was developed by the Consortium for the Advanced Simulation of LWRs (CASL), employs for the radiation transport portion a many-group, 2-D/1D method, where the 2-D radial planes are solved using the Method of Characteristics and 1D axial direction solved using an SPN method. So radially the same method is employed as in many lattice physic codes, and axially some compromise is made in using an SPN approximation. Depletion is done employing ORIGEN, where about 250 isotopes are tracked, and thermal-hydraulics is simulated utilizing CTF [3], which employs a subchannel, two-fluid, and three-field model. Regarding NESTLE, for this work two-groups are employed and the nodal solve is done using a quadric polynomial, with pin power reconstruction done first determining the intra-nodal flux and fission cross-section distributions followed by application of pin-power form factors [4]. The next section provides furthers details as related to how the nodal parameters have been determined, followed by a section contrasting VERA with NESTLE predictions, concluding with a section presenting conclusions.

\section{GENERATION AND FUNCTIONALIZATION OF NODAL PARAMETERS}

\subsection{Generation of Nodal Parameters}

To provide the information needed to generate the nodal parameters, the core simulator component of VERA was executed for a number of different state points, differing in core power level, coolant inlet temperature, and soluble boron concentration. VERA code modifications were made to determine and edit out the following calculated nodal quantities based upon $2 \times 2$ nodalization per lattice, the original VERA axial mesh, and if applicable 2 energy groups: node average flux, 6 surfaces average currents and fluxes, 8 corner points fluxes, macroscopic cross-sections for various reaction types, microscopic 
absorption cross-sections and number densities for transient fission products, neutron velocity, pin powers for each axial mesh span, fuel surface and volume temperatures, coolant temperature and density, soluble boron concentration, delayed neutron fractions, rodded state, wet fraction, and burnup. In addition, absolute value of core average flux, core geometry, loading pattern, and other VERA pass-through information was written out to facilitate the automated preparation of NESTLE input files. To generate the nodal cross-sections, which will be characterized by thermal-hydraulics conditions, control state, burnup and location, VERA utilized the well know formulation

$$
\Sigma_{x_{g}}^{n}=\frac{\int_{\Delta V_{n}} \int_{E_{g-1}}^{E_{g}} d V d E \Sigma_{x}^{n}(\vec{r}, E) \Phi(\vec{r}, \mathrm{E})}{\int_{\Delta V_{n}} \int_{E_{g-1}}^{E_{g}} d V d E \Phi(\vec{r}, \mathrm{E})}
$$

where the notation is standard, with $x$ indicating the reaction type, $g$ the few energy groups, $n$ the spatial node, and $\Phi$ the VERA predicted scalar flux. In reality, the integrals are replaced by summations over the fine spatial mesh of the depletion zones and fine energy groups utilized in VERA. Note that in contrast to the lattice physics methodology which produces homogenized cross-sections in terms of lattice type (color), VERA will produce them in terms of spatial node position, i.e. on a node-by-node basis, accounting for all of the effects of T-H feedback, history and neutron spectrum, including effects of neighboring nodes. To avoid the need for current users of NESTLE to install a modified version of the code, a color will be assigned to every spatial node avoiding the need for code modifications.

The VINO (VERA In - Nodal Out) code was written to process the information output by VERA and prepare some of the NESTLE input files. Capability to either further radially homogenize, e.g. 1x1 mesh as used in training simulators, or energy groups collapse (1 group) exist in VINO but were not employed for the work reported herein. However, the capability to axially homogenize was employed for one of the cases analyzed. Since non-zero boundary conditions are now being employed, a variation of the traditional manner of obtaining the DF [5] is required to preserve the nodal surface average currents for a state point. Suppose that a 3-D one-node problem solution capability exists, which utilizes exactly the same solution methodology as employed in the nodal core simulator, which for NESTLE employs a quartic representation for the 1-D transverse flux and quadratic representation of the transverse leakage. This one node problem can be solved by employing the nodal cross-sections, node average flux, and nodal surface average currents on one $\mathrm{x}, \mathrm{y}$ and $\mathrm{z}$ surface as predicted by VERA. By neutron balance over the node, the nodal surface average currents on the other $\mathrm{x}, \mathrm{y}$ and $\mathrm{z}$ surfaces is assured to be preserved, i.e. equal the values predicted by VERA. There is one exception to this, and that being for nodes involving certain boundary condition. For nodes whose boundary condition is either zero flux or zero reentrant current, the diffusion theory approximation used in nodal codes when used to impose a surface boundary condition, will not exactly preserve the nodal surface average current on the opposite surface. Numerical tests have shown that the difference in this current's value between VERA and the one-node solution is very small, so judged an acceptable error. From the transverse flux solution the 'homogeneous' surface average flux can be obtained on each surface, which in turn enables the DF to be obtained as indicated,

$$
D F_{g}^{n, 1}=\frac{\int_{\Delta S_{n, l}} \int_{E_{g-1}}^{E_{g}} d S d E \Phi(\vec{r}, \mathrm{E})}{\int_{\Delta S_{n, l}} \int_{E_{g-1}}^{E_{g}} d S d E \tilde{\Phi}(\vec{r}, \mathrm{E})}
$$


where $l$ denotes the nodal surface, $\Phi$ the VERA predicted 'heterogeneous' flux, and $\tilde{\Phi}$ the nodal predicted 'homogeneous' flux. Note that in contrast to the lattice physics methodology, where DF are independent of the nodal method subsequently employed, the proposed methodology produces DF that are nodal method dependent since the 3-D one-node problem solution method employs the nodal method of the nodal core simulator. Specifically for NESTLE, the nodal method employs a quartic polynomial for flux and quadratic polynomial for transverse leakage when solving the 1-D transverse integrated diffusion equation. Also note that DF are determined for the axial direction, which is not the case when using a lattice physics code.

Key remaining nodal nuclear parameters that are required are the corner DF and PFF [5]. The form factors are utilized in NESTLE to obtain the pin powers contained within the node by using the following equation,

$$
\hat{p}^{n, p}(z)=P F F^{n, p}(z)\left(\hat{p}^{n, p}\right)^{\text {Intra }}
$$

where $p$ denotes the pin; $\hat{p}^{n, p}(z)$ the reconstructed pin power; $P F F^{n, p}(z)$ the PFF; and $\left(\hat{p}^{n, p}\right)^{\text {Intra }}$ the intra-nodal pin power. The intra-nodal pin power, which captures the smooth variation of the power within the node, attempts to correct for the variation in the PFF from the reference state to the core state NESTLE is analyzing. The difference when contrasted to a lattice physics code approach to obtaining the PFF is that since VERA predicted pin powers will mostly capture the within node flux variation due to history, control rod insertion, boundary conditions and local thermal-hydraulics feedback effects, any correction imposed by the intra-nodal flux would be expected to be small.

The corner DF, employed in determining the intra-nodal flux, are obtained in a similar fashion to the surface DF, but now evaluated at node corners using the one-node solution. To determine the intra-nodal power from the intra-nodal flux, values of the intra-nodal $\kappa \Sigma_{f}$ are required. Since the pin power form factors contain all the history and thermal-hydraulics feedback effects on a pin-by-pin basis, the intranodal $\kappa \Sigma_{f}$ can now be approximated by the node average value versus utilizing a smoothly varying spatially function fit to surface and node average values. To determine the PFF, here again a modification from the methodology used in lattice physics codes is required due to the non-zero boundary condition associated with the VERA solution. One can rearrange the equation to solve for the PFF if one knew the pin powers and intra-nodal flux. With the pin powers predicted by VERA, the task to complete in VINO is to predict the intra-nodal flux. To solve for the intra-nodal flux, the NESTLE based functionalization requires the node average flux, surface average 'homogeneous' fluxes, and corner point 'homogeneous' fluxes to be known. These quantities can all be evaluated from the one-node solution completed to determine the DF. Hence the PFF are determined using

$$
\operatorname{PFF}^{n, p}(z)=p^{n, p}(z) /\left(p^{n, p}\right)^{\text {Intra }}
$$

where $p^{n, p}(z)$ is the VERA determined pin 'heterogeneous' powers; and $\left(p^{n, p}\right)^{\text {Intra }}$ is the intra-nodal pin 'homogeneous' powers for the reference state point determined from the one-node solution. Do note that the corner DF and intra-nodal powers are axially integrated within the node. This formulation equates to assuming that the intra-nodal flux has no axial dependence within a node, which is a reasonable assumption except in the vicinity of a partial control rod insertion. Indeed, results obtained showed how spacer grid induced power depressions as predicted by a VERA fine axial mesh model are preserved with the just noted within-node, axial dependent form factors. Also note that if $\left(\hat{p}^{n, p}\right)^{\text {Intra }} \propto\left(p^{n, p}\right)^{\text {Intra }}$, which 
would be expected except for unusual core conditions, the reconstructed pin powers would assume the fine pin structure obtained from VERA. Conforming to the current practice used in NESTLE, surface and corner DF and PFF are not functionalized in terms of thermal-hydraulic and soluble poison concentration conditions, but are dependent upon rodded state.

One additional attribute that needs to be determined from VERA output is the fission products' yields. To assure that the number densities for Pm-149, I-135 and Xe-135 are preserved from node average values predicted by VERA, which has assumed equilibrium conditions for these transient fission products, an inverse calculation is completed. That is, having calculated the node average quantities for the microscopic cross-sections and isotopic number densities for these fission products, and the flux, the relevant, equilibrium Bateman equations can be rearranged such that the unknowns are now fission product yields in contrast to isotopic number densities. This is the approach that has been employed, producing fission product yields that are fuel color (i.e. spatial position) dependent. Indeed, they are also $\mathrm{T}-\mathrm{H}$ conditions, soluble poison concentration, and rodded state dependent, with all of these dependencies for now ignored in the processing.

\subsection{Functionalization of Nodal Parameters}

Some of the nodal nuclear parameters, specifically the macroscopic and microscopic cross-sections, will be functionalized in terms of node T-H conditions, burnup, soluble boron concentration, and rodded state. To capture depletion effects, the VERA depletion calculation will need to be completed consistent with expected, actual or bounding plant operations, depending upon the application. By doing this there is no need to utilize an approach to correct for history effects, since VERA captures all these effects and reflects them in the nuclear parameters it generates. Exactly how the nodal nuclear parameters will be functionalized will now be described.

The NINO (Nodal In - NESTLE Out) code was written to read the VINO generated output containing the values of the nodal parameters, complete functional fits to the nodal parameters, and writing the input files required for NESTLE. The functionalization is based upon one of the available options in NESTLE:

$$
\Sigma\left(\rho_{c}, T_{f}, N_{p}\right)=\Sigma_{o}+\sum_{i=1}^{2} a_{i}\left(\rho_{c}-\rho_{\mathrm{o}}\right)^{i}+c\left(\sqrt{T_{f}}-\sqrt{T_{f_{\mathrm{o}}}}\right)+\sum_{j=1}^{2} d_{j}\left(N_{p}-N_{p_{\mathrm{o}}}\right)^{j}
$$

To determine the fitting coefficients of this equation requires a minimum of six executions of VERA, where three coolant densities $\left(\rho_{c}\right)$, two fuel temperatures $\left(T_{f}\right)$, and three soluble poison concentrations $\left(N_{p}\right)$ values must appear in the state points. Each of the VERA state points is processed by VINO, with the VINO nodal parameter outputs for all the state points then read into NINO. With each node having a distinct set of nodal parameters, the associated nodal values of $\left(\rho_{c}, T_{f}, N_{p}\right)$ as determined by VERA are used in the fitting.

The fitting coefficients are determined by NINO via a least squares fit, since there may be more state points than the minimum required, using the LAPACK SGELS and supporting subroutines. All macroscopic and transient fission products' microscopic cross-sections are fitted. As noted, surface and corner DF, and PFF are not fitted and set to their values for a specified state point. Fits are completed for all nodes in the unrodded state, with a separate fit completed for nodes which could be in a rodded state. Note the non-uniqueness now of generating the unrodded and rodded nodal parameters, since an unrodded node's parameters could be obtained from either a totally unrodded or partially rodded VERA state point. Likewise, a rodded node's parameters could be obtained from different partially rodded VERA state points. This ambiguity does not arise in lattice physics calculations since full core conditions 
are not captured. Burnup dependence is to be treated by completing depletion using VERA, followed by at selected depletion steps VERA branch case state point executions, VINO and NINO processing of VERA outputs, and finally NESTLE interpolation of functionalized nodal parameters.

\section{AGREEMENT BETWEEN VERA AND NESTLE PREDICITONS}

\subsection{Generation of Nodal Parameters}

A core model of a four-loop Westinghouse Generation II pressurized water reactor [6] was employed to compare VERA and NESTLE predictions of core reactivity and power distribution. Core models employed both 58/24 and 2/2 VERA/NESTLE axial mesh models, the former requiring axial collapse and used for VERA and VINO verification and the later used for VERA, VINO and NINO verification. Table I indicates the state point values employed, chosen with minimum consideration other than satisfying the minimum number of state point values required for nodal parameters' fitting. VERA was executed for all the state points for both unrodded and full inserted rodded states, with relevant output processed by VINO and NINO. Note that there are more state points than the minimum required in completing the functionalization. When using only six of these state points (Cases 5-10), exact fits to nodal parameters were obtained as expected. When using all ten state points, the least square fits produced very small fitting errors when evaluated as the state point conditions (e.g. maximum relative error below 0.0015). NESTLE was then executed for these state points. VERA predicted nodal values of $\left(\rho_{c}, T_{f}, N_{p}\right)$ were input to NESTLE to isolate differences in predictions to the neutronics component. Prior to using the functionalized nodal parameters, for VERA and VINO verification the nodal parameters for a single state point were directly used by NESTLE for the finer axial mesh models. Nearly perfect agreements between VERA and NESTLE predictions are to be expected, confirmed by differences of $1 \mathrm{pcm}$ in core reactivity and maximum difference of 0.002 in pin relative powers.

Table I. State point values used in generating nodal parameters and assessing NESTLE accuracy.

\begin{tabular}{|c|c|c|c|}
\hline State & $\begin{array}{c}\text { Power } \\
(\% \text { Rated })\end{array}$ & $\begin{array}{c}\text { Inlet } \\
\text { Temperature } \\
(\mathrm{K})\end{array}$ & $\begin{array}{c}\text { Boron } \\
\text { Concentration } \\
(\mathrm{ppm})\end{array}$ \\
\hline 1 & 0 & 565 & 1286.96 \\
\hline 2 & 25 & 565 & 1134.14 \\
\hline 3 & 50 & 565 & 1062.17 \\
\hline 4 & 75 & 565 & 1015.79 \\
\hline 5 & 118 & 565 & 959.68 \\
\hline 6 & 100 & 565 & 980.88 \\
\hline 7 & 100 & 575 & 980.88 \\
\hline 8 & 100 & 555 & 980.88 \\
\hline 9 & 100 & 565 & 961.59 \\
\hline 10 & 100 & 565 & 1000.27 \\
\hline
\end{tabular}

Next the agreement utilizing functionalized cross-sections was completed for the 2 axial mesh model. Core rodded conditions considered included ARO, Control Bank D fully inserted (CD), and Control Bank D fully inserted with the core's center control rod ejected (CD-1). Functionalization was first completed using six VERA state points, so exact fits are to be expected and were observed. Focusing on CD-1 for Case 10, the reactivity difference between VERA and NESTLE was $10 \mathrm{pcm}$, but maximum differences in assembly power (0.02) and pin power (0.04) were larger than desired as shown in Figure 1. A radial tilt is to be noted in the assembly power distribution. This was traced to the usage of unrodded nodal parameters 
generated using the ARO VERA state points (accounting for $\sim 75 \%$ of tilt), analogous to what would be done using a lattice physics approach. In addition, the usage of ARO VERA state points cross-sections' fits for unrodded nodes required extrapolation to T-H conditions beyond the fitted range (accounting for $\sim 25 \%$ of tilt). For the differences in pin powers, large for pins in assemblies adjacent to the rodded assemblies, this is mainly attributed to the usage of corner DF and PFF generated using the ARO VERA state points. When obtaining the unrodded parameters from the CD-1 VERA state points, the maximum reactivity difference decreased to $2 \mathrm{pcm}$ and excellent agreement in assembly power $(0.003)$ and pin power $(0.003)$ was obtained as shown in Figure 1. The implication is that a rodded neighboring assembly noticeable affects the unrodded assemblies' nodal parameters. What contributions cross-sections, surface and corner DF, and PFF each make to inaccuracies remains to be evaluated. Next the fitting was completed using the ten VERA state points where exact fitting to the state point values is no longer possible. The differences in core reactivity and pin powers were nearly the same as observed when using six VERA state points (i.e. exact fits), which is to be expected since fitting errors of cross-sections were small as previously noted.
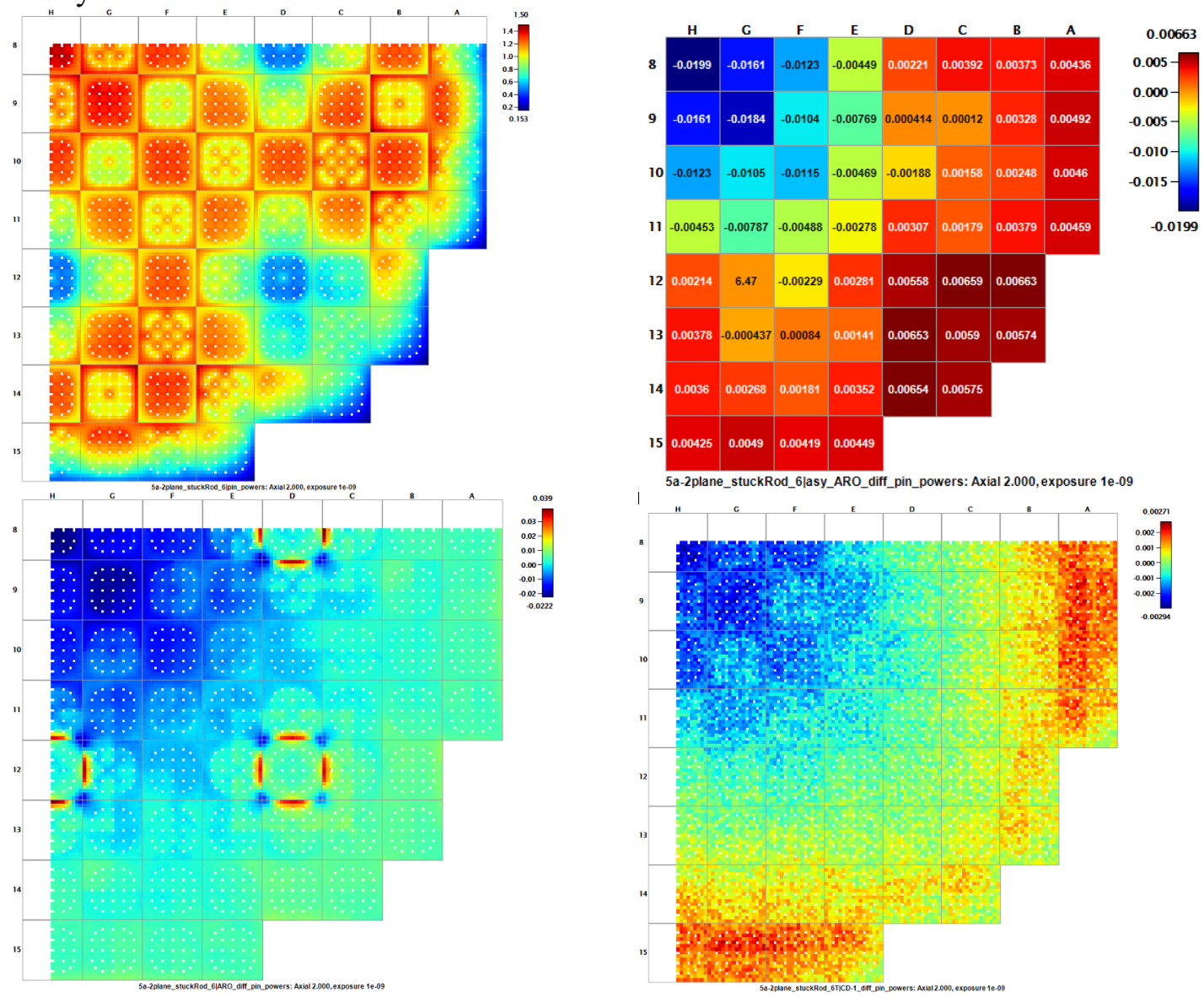

Figure 1. Case 10 CD-1 VERA pin power distribution (upper left) and NESTLE - VERA assembly power differences (upper right) using VERA ARO unrodded nodal parameters state points, and NESTLE - VERA pin power distribution generated using VERA ARO unrodded nodal parameters (bottom left) and VERA CD-1 (bottom right) state points [note scale change for these two figures].

Next the agreement in core reactivity and powers for a number of state points was completed, based upon using ten state points for the fitting and initially the VERA ARO state points to generate the unrodded nodal parameters. For the ARO state points, reactivity and power differences were very small as to be expected, given the small errors in cross-section fits. As seen for Case $10 \mathrm{CD}-1$ state point, the differences for the rodded state points were larger, with the reactivity difference for Case $1 \mathrm{CD}-1$ being $58 \mathrm{pcm}$. This 
is again mainly attributed to the usage of unrodded nodal parameters generated using the VERA ARO state points. When using the VERA CD-1 state points to fit both the unrodded and rodded nodal parameters, the maximum difference in reactivity is reduced to $2 \mathrm{pcm}$ for Case $1 \mathrm{CD}-1$. Similar behaviors for the assembly and pin powers are observed.

\section{CONCLUSIONS}

The capability to utilize a high fidelity core simulator (VERA) in support of determining nodal parameters has been demonstrated with regard to producing accurate predictions of core reactivity and pin power distribution from a nodal code (NESTLE). Most assumptions associated with employing a lattice physics code have been eliminated using this approach. In this fashion, it is anticipated that the accuracy of a high fidelity core simulator can be obtained but without the associated computer resource and wall clock time requirements. This would be most beneficial when considering transient simulations and for real time applications. Unacceptable large differences between VERA and NESTLE in core reactivity, and assembly and pin powers, when the core is partially rodded, were observed, attributed to the usage of ARO VERA state points in determining the unrodded nodal parameters. By usage of the associated rodded VERA state points in generating the unrodded nodal parameters, these differences were reduced to being very small. Additional verification is in progress, including considering agreement at non-fitted state point conditions and partially inserted control rods. As a word of caution, the conclusions reached in this study are applicable to the methodologies employed by NESTLE but may not be applicable to other methodologies used in nodal core simulators.

\section{ACKNOWLEDGMENTS}

This research was supported by the U.S. Department of Energy under Contract DE-SC0018915, and used resources of the Compute and Data Environment for Science (CADES) at the Oak Ridge National Laboratory, which is supported by the Office of Science of the U.S. Department of Energy under Contract No. DE-AC05-00OR22725.

\section{REFERENCES}

1. B. Kochunas, B. Collins, S. Stimpson, R. Salko, D. Jabaay, A. Graham, Y. Liu, K. S. Kim, W. Wieselquist, A. Godfrey, K. Clarno, S. Palmtag, T. Downar and J. Gehin, "VERA Core Simulator Methodology for Pressurized Water Reactor Cycle Depletion," Nuclear Science and Engineering, 185 (2017) .

2. P. J. Turinsky, R. M. K. Al-Chalabi, P. Engrand, H. N. Sarsour, F. X. Faure and W. Guo, NESTLE: A Few-Group Neutron Diffusion Equation Solver Utilizing the Nodal Expansion Method for Eigenvalue, Adjoint, Fixed-Source Steady State and Transient Problems, EGG-NRE-11406, INEL (1994).

3. R. Salko, M. Avramova, A. Wysocki, A. Toptan, J. Hu, N. Porter, T. Blyth, C. Dances, A. Gomez, C. Jernigan, and J. Kelly, CTF 4.0 Theory Manual, CASL Publication, ORNL (2019).

4. S. Palmtag, K.S. Smith, and A.F. Henry, "An Advanced Nodal Method with Pin Power Reconstruction for MOX Fuel Calculations," Proc. ANS Topical Meeting - Advances in Nuclear Fuel Management II, ANS, Myrtle Beach (1997).

5. K.S. Smith, Spatial Homogenization Methods for Light Water Reactors, Doctorate Dissertation, MIT (1980).

6. A.T. Godfrey, B.S. Collins, K.S. Kim, R. Lee, J. Powers, R. Salko, S.G. Stimpson, W.A. Wieselquist, R. Montgomery, R. Montgomery, B. Kochunas, D.R. Jabaay, N. Capps and J. Secker, VERA Benchmarking Results For Watts Bar Nuclear Plant, CASL Technical Report: CASL-U-2015-0206000, ORNL (2015). 